\title{
Spin polarization dynamics in the Gubser-expanding background
}

\author{
Rajeev Singh $\oplus^{*}$ Gabriel Sophys $\odot,{ }^{\dagger}$ and Radoslaw Ryblewski ${ }^{\ddagger}$ \\ Institute of Nuclear Physics Polish Academy of Sciences, PL 31-342 Kraków, Poland
}

(Received 8 December 2020; accepted 24 March 2021; published 27 April 2021)

\begin{abstract}
Evolution of spin polarization in the Gubser-expanding conformal perfect-fluid hydrodynamic background is studied. The analysis of the conformal transformation properties of the conservation laws is extended to the case of the angular momentum conservation. The explicit forms of equations of motion for spin components are derived and analyzed, and some special solutions are found.
\end{abstract}

DOI: 10.1103/PhysRevD.103.074024

\section{INTRODUCTION}

In the last decades, relativistic hydrodynamics has proven to be very successful in describing the evolution of the strongly interacting matter produced in relativistic heavy-ion collisions [1-8]. Recent measurements of spin polarization of $\Lambda$ hyperons have shown that the spacetime evolution of the quantum spin should also be included in this framework in order to properly capture effects seen in the experiment [9-15].

The first attempt to formulate relativistic hydrodynamics with spin has been done in Ref. [16]; see also the follow-up papers [17-24], as well as reviews [25,26]. In contrast to previous theoretical studies which considered the generation of spin polarization of matter at freeze-out due to spin-vorticity coupling [27-47] (see also related studies [48-89]), the spin hydrodynamic approach has been formulated entirely based on the conservation laws and assumption of local thermal equilibrium. In this case, enforcement of total angular momentum conservation leads to the need for introducing additional Lagrange multipliers which comprise the so-called spin polarization tensor $\omega^{\mu \nu}$ that, in the case of global equilibrium with rigid rotation, reduces to the thermal vorticity tensor $\varpi_{\mu \nu}=-\frac{1}{2}\left(\partial_{\mu} \beta_{\nu}-\right.$ $\partial_{\nu} \beta_{\mu}$ ) (here $\beta_{\mu}=U_{\mu} / T$ is the ratio of the fluid flow vector $U_{\mu}$ and the local temperature $T$ ) [90-92]. The resulting spin hydrodynamic framework extends the standard one by adding additional dynamic equations which, in general, have to be solved numerically.

\footnotetext{
*rajeev.singh@ifj.edu.pl $\dagger$ gabriel.sophys@ifj.edu.pl

*radoslaw.ryblewski@ifj.edu.pl
}

Published by the American Physical Society under the terms of the Creative Commons Attribution 4.0 International license. Further distribution of this work must maintain attribution to the author(s) and the published article's title, journal citation, and DOI. Funded by SCOAP ${ }^{3}$.
In the present paper we use the formalism of relativistic hydrodynamics with spin proposed in Refs. [22,25] to study the evolution of spin polarization in the Gubserexpanding perfect-fluid hydrodynamic background [93,94]. The current work is an extension of the study presented in Ref. [22] where the spacetime evolution of the spin polarization was considered in a boost-invariant and transversely homogeneous background, also known as Bjorken flow. Following other works [95-104] we first solve the perfectfluid hydrodynamical equations using the Gubser symmetry arguments in the de Sitter coordinates and obtain analytical solutions for hydrodynamic variables. Subsequently, we extend the analysis of the properties of the conservation laws with respect to the conformal transformation to the case of the angular momentum conservation. We find that the latter is conformally invariant only if the spin tensor is antisymmetric in all indices and the particles composing the fluid are massless. As the de Groot-van Leeuwen-van Weert (GLW) spin tensor [105] considered in this work is not, in general, satisfying these requirements, we relax the constraints related to the symmetry with respect to the special conformal transformations exploiting only the cylindrical symmetry and boost invariance. Using expressions for temperature, chemical potential, and velocity obtained for the Gubser-flowing background, we derive the equations of motion for spin polarization components which, as expected, exhibit nontrivial dynamics in both de Sitter time and angle, as well as a (small) sensitivity to the mass of the constituent particles. As in the formulation used herein, we keep the assumption of a small amplitude of polarization effects $[22,25]$, the background equations expressing the baryon charge and energy-momentum conservation decouple completely from the angular momentum conservation, and the background solutions are not spoiled by the breaking of the symmetry at the level of angular momentum conservation. For the special case of massless particles, we find a set of special solutions which, in general, have a powerlike dependence on the temperature of the system. Finally, we also present some full numerical solutions with finite masses 
in Milne spacetime. The solutions obtained in this work may be used for describing the collisions of systems with initial spin polarization as well as for testing the full $3+1 \mathrm{D}$ geometry codes.

The structure of the paper is as follows: We start by briefly reviewing the framework of relativistic hydrodynamics with spin for polarized systems of spin $-\frac{1}{2}$ particles in Sec. III. In Sec. IV we introduce the boost invariance and cylindrical symmetry, which is followed in Sec. V by the details about Gubser symmetry, Weyl rescaling of various thermodynamical and hydrodynamical quantities, and the information about the de Sitter basis vectors. Conformal transformation of the conservation equations is studied in Sec. VI. In Sec. VII we derive the Gubser-symmetric evolution equations for the background which we then use to find the evolution equations of spin polarization components in the de Sitter spacetime. The resulting equations of motion are studied both analytically and numerically and transformed back to Milne spacetime. We conclude and summarize in Sec. VIII. Some additional information and details are given in the Appendixes.

\section{CONVENTIONS}

We use the following shorthand notation for the scalar product between two four-vectors: $a_{\mu} b^{\mu} \equiv a \cdot b$. For the Levi-Civita tensor $\epsilon^{\alpha \beta \gamma \delta}$, the sign convention used throughout the paper is $\epsilon^{0123}=-\epsilon_{0123}=1$. Throughout the text we use natural units with $c=\hbar=k_{B}=1$. The antisymmetrization of arbitrary rank-two tensor $A$ is denoted as $A_{[\mu \nu]}=\frac{1}{2 !}\left(A_{\mu \nu}-A_{\nu \mu}\right)$. Also, we are using "mostly plus" metric signature.

\section{PERFECT-FLUID HYDRODYNAMICS FOR SYSTEMS OF PARTICLES WITH SPIN 1/2}

For the reader's convenience, in this section we briefly review the recently developed formalism of relativistic perfect-fluid hydrodynamics for polarized systems of particles with spin $\frac{1}{2}$ [22]. We use the approximation of small polarization which, in the leading order, leads to decoupling of the background hydrodynamic equations for velocity, temperature, and chemical potential (given by the standard net baryon number and energy-momentum conservation laws) from the dynamics of spin degrees of freedom (resulting from the conservation of angular momentum).

\section{A. Conservation of net baryon number}

The conservation law of the net baryon number can be expressed in the following way:

$$
d_{\alpha} N^{\alpha}(x)=0,
$$

where, in the case of no dissipative effects, the net baryon current $N^{\alpha}$ reads

$$
N^{\alpha}=\mathcal{N} U^{\alpha},
$$

with the net baryon density being given by

$$
\mathcal{N}=4 \sinh \alpha \mathcal{N}_{(0)} .
$$

The fluid four-velocity $U^{\alpha}$ in Eq. (2) is normalized as $U \cdot U=-1$ and $d$ denotes the covariant derivative (see Appendix A for more information on the definitions of covariant derivative). The quantity $\alpha$ is the ratio of baryon chemical potential $\mu$ and temperature $T, \alpha \equiv \mu / T$. For an ideal relativistic gas of classical massive particles (and antiparticles) the auxiliary number density $\mathcal{N}_{(0)}$ is given by the well-known expression [1]

$$
\mathcal{N}_{(0)}=k T^{3} z^{2} K_{2}(z),
$$

where $z \equiv m / T$ with $m$ being the mass of the particles (and antiparticles), $k \equiv 1 /\left(2 \pi^{2}\right)$. and $K_{n}$ denotes the $n$th modified Bessel function of the second kind.

\section{B. Conservation of energy and linear momentum}

The conservation of energy and linear momentum is given by the expression

$$
d_{\alpha} T^{\alpha \beta}(x)=0,
$$

where, for perfect fluid, the energy-momentum tensor $T^{\alpha \beta}$ takes the following standard form:

$$
T^{\alpha \beta}=\mathcal{E} U^{\alpha} U^{\beta}+\mathcal{P} \Delta^{\alpha \beta},
$$

with $\Delta^{\alpha \beta} \equiv g^{\alpha \beta}+U^{\alpha} U^{\beta}$ being the spatial projection operator orthogonal to the fluid flow vector and the energy density and pressure being defined as

$$
\begin{aligned}
\mathcal{E} & =4 \cosh \alpha \mathcal{E}_{(0)}, \\
\mathcal{P} & =4 \cosh \alpha \mathcal{P}_{(0)},
\end{aligned}
$$

respectively. In the case of ideal relativistic gas of spinless and neutral massive Boltzmann particles. the auxiliary energy density and pressure are [1]

$$
\begin{gathered}
\mathcal{E}_{(0)}=k T^{4} z^{2}\left[z K_{1}(z)+3 K_{2}(z)\right], \\
\mathcal{P}_{(0)}=T \mathcal{N}_{(0)},
\end{gathered}
$$

respectively. The thermodynamic quantities defined above satisfy the standard thermodynamic relations, namely,

$$
\begin{aligned}
& \mathcal{E}+\mathcal{P}=T \mathcal{S}+\mu \mathcal{N}, \\
& \mathcal{E}_{(0)}+\mathcal{P}_{(0)}=T \mathcal{S}_{(0)},
\end{aligned}
$$

with $\mathcal{S}=\left(\frac{\partial \mathcal{P}}{\partial T}\right)_{\mu}, \mathcal{N}=\left(\frac{\partial \mathcal{P}}{\partial \mu}\right)_{T}$, and $\mathcal{S}_{(0)}=\frac{\partial \mathcal{P}_{(0)}}{\partial T}$. 


\section{Conservation of angular momentum}

Total angular momentum may be expressed as a sum of the orbital angular momentum part $L^{\alpha, \beta \gamma}$ and spin angular momentum part $S^{\alpha, \beta \gamma}$ as follows:

$$
J^{\alpha, \beta \gamma}=L^{\alpha, \beta \gamma}+S^{\alpha, \beta \gamma}=x^{\beta} T^{\alpha \gamma}-x^{\gamma} T^{\alpha \beta}+S^{\alpha, \beta \gamma},
$$

where, in the second equality, we expressed the orbital part in terms of the energy-momentum tensor. The conservation of the total angular momentum is given by the expression

$$
d_{\alpha} J^{\alpha, \beta \gamma}=d_{\alpha} S^{\alpha, \beta \gamma}+2 T^{[\beta \gamma]}=0 .
$$

Since the energy-momentum tensor used herein is symmetric $T^{[\beta \gamma]}=0$ [see Eq. (6)], the conservation of the total angular momentum implies separate conservation of its spin part.

The spin tensor in Eq. (14) is given by the following expression [22]:

$$
S^{\alpha, \beta \gamma}=\mathcal{C} U^{\alpha} \omega^{\beta \gamma}+S_{\Delta}^{\alpha, \beta \gamma},
$$

where the auxiliary spin tensor is defined as

$$
\begin{aligned}
S_{\Delta}^{\alpha, \beta \gamma}= & \mathcal{A} U^{\alpha} U^{\delta} U^{[\beta} \omega^{\gamma]}{ }_{\delta} \\
& +\mathcal{B}\left(U^{[\beta} \Delta^{\alpha \delta} \omega^{\gamma]}+U^{\alpha} \Delta^{\delta[\beta} \omega^{\gamma]}+U^{\delta} \Delta^{\alpha[\beta} \omega^{\gamma]}\right),
\end{aligned}
$$

with the quantity $\omega^{\alpha \beta}(x)$ being the spin polarization tensor (to be discussed in the next section). The thermodynamic quantities $\mathcal{A}, \mathcal{B}$, and $\mathcal{C}$ in Eqs. (15) and (16) are

$$
\begin{gathered}
\mathcal{A}=2 \mathcal{C}-3 \mathcal{B}, \\
\mathcal{B}=-\frac{\mathcal{E}+\mathcal{P}}{2 T z^{2}}, \\
\mathcal{C}=\frac{\mathcal{P}}{4 T} .
\end{gathered}
$$

In what follows, here onwards we will refer to Eqs. (15) and (16) as the GLW spin tensor [105].

\section{Spin polarization tensor}

The spin polarization tensor $\omega^{\alpha \beta}$ is an asymmetric ranktwo tensor which can be decomposed with respect to the fluid four-velocity in the following way:

$$
\omega^{\alpha \beta}=\kappa^{\alpha} U^{\beta}-\kappa^{\beta} U^{\alpha}+\epsilon^{\alpha \beta \gamma \delta} U_{\gamma} \omega_{\delta} .
$$

Any part of the auxiliary four-vectors $\kappa^{\alpha}$ and $\omega^{\alpha}$ parallel to $U^{\alpha}$ does not contribute to the right-hand side of Eq. (20) hence, without the loss of generality, we can assume that $\kappa^{\alpha}$ and $\omega^{\alpha}$ satisfy the following orthogonality conditions:

$$
\kappa \cdot U=0, \quad \omega \cdot U=0 .
$$

Using the above conditions, $\kappa_{\alpha}$ and $\omega_{\alpha}$ can be expressed in terms of the spin polarization tensor $\omega_{\alpha \beta}$ as follows:

$$
\kappa_{\alpha}=\omega_{\alpha \beta} U^{\beta}, \quad \omega_{\alpha}=\frac{1}{2} \epsilon_{\alpha \beta \gamma \delta} \omega^{\beta \gamma} U^{\delta} .
$$

\section{IMPLEMENTATION OF BOOST INVARIANCE AND CYLINDRICAL SYMMETRY}

Let us consider central high-energy heavy-ion collisions which are boost invariant and cylindrically symmetric with respect to the beam $(z)$ axis. Their dynamics is most conveniently described in the polar-hyperbolic coordinates $x^{\mu}=(\tau, r, \phi, \eta)$ where the line element reads

$$
d s^{2}=-d \tau^{2}+d r^{2}+r^{2} d \phi^{2}+\tau^{2} d \eta^{2},
$$

with $\tau=\sqrt{t^{2}-z^{2}}$ being the longitudinal proper time, $\eta=$ $\tanh ^{-1}(z / t)$ being the longitudinal spacetime rapidity, and $r=\sqrt{x^{2}+y^{2}}$ and $\phi=\tan ^{-1}(y / x)$ denoting the radial distance and the azimuthal angle, respectively, parametrizing the transverse plane perpendicular to the beam direction.

For convenience. we introduce the following orthogonal four-vector basis in the laboratory frame:

$$
\begin{aligned}
U^{\mu} & =(\cosh \vartheta, \sinh \vartheta, 0,0), \\
R^{\mu} & =(\sinh \vartheta, \cosh \vartheta, 0,0), \\
\Phi^{\mu} & =(0,0,1 / r, 0), \\
Z^{\mu} & =(0,0,0,1 / \tau),
\end{aligned}
$$

which allows us to express the polar-hyperbolic metric tensor, $g^{\mu \nu}=\operatorname{diag}\left(-1,1,1 / r^{2}, 1 / \tau^{2}\right)$, in the following form:

$$
g^{\mu \nu}=-U^{\mu} U^{\nu}+R^{\mu} R^{\nu}+\Phi^{\mu} \Phi^{\nu}+Z^{\mu} Z^{\nu} .
$$

It is straightforward to check that the basis vectors (24) satisfy the following normalization conditions:

$$
\begin{aligned}
& U \cdot U=-1, \quad R \cdot R=1, \\
& \Phi \cdot \Phi=1, \quad Z \cdot Z=1,
\end{aligned}
$$

while all mixed scalar products vanish. One can convince himself that in the local rest frame $U, R, \Phi$, and $Z$ are the unit vectors pointing in the $\tau, r, \phi$, and $\eta$ directions, respectively.

Using the basis vectors (24) and orthogonality conditions (21), four-vectors $\kappa^{\alpha}$ and $\omega^{\alpha}$ may be decomposed as follows: 


$$
\begin{aligned}
& \kappa^{\alpha}=a_{R} R^{\alpha}+a_{\Phi} \Phi^{\alpha}+a_{Z} Z^{\alpha}, \\
& \omega^{\alpha}=b_{R} R^{\alpha}+b_{\Phi} \Phi^{\alpha}+b_{Z} Z^{\alpha},
\end{aligned}
$$

where $a_{i}(\tau, r)$ and $b_{i}(\tau, r)$ are scalar coefficients characterizing the components of the spin polarization tensor along the basis vectors.

\section{GUBSER SYMMETRY AND CONFORMAL MAPPING TO DE SITTER SPACE}

For boost-invariant and cylindrically symmetric systems, one can construct a nontrivial four-velocity profile in Minkowski space $R^{3} \otimes R$ which is invariant with respect to the $S O(3)_{q} \otimes S O(1,1) \otimes Z_{2}$ conformal symmetry group, also known as "Gubser's symmetry" [93,94]. The latter is composed of rotations in the $r-\phi$ plane coupled with two special conformal transformations parametrized by an arbitrary inverse length scale $q\left[S O(3)_{q}\right]$, boosts in the $\eta$ direction $[S O(1,1)]$, and reflections with respect to the $r-\phi$ plane $\left(Z_{2}\right)$. The procedure of finding the flow pattern invariant with respect to this symmetry, while being intricate in Minkowski space, is quite transparent when considered in the curved spacetime formed by the product of the three-dimensional de Sitter space and a line, $d S_{3} \otimes R$-hereafter we will shortly refer to it as "de Sitter space." The conformal map used to transform from one to the other constitutes the Weyl rescaling of the line element

$$
d s^{2} \rightarrow \frac{d s^{2}}{\Omega^{2}}=\frac{-d \tau^{2}+d r^{2}+r^{2} d \phi^{2}}{\tau^{2}}+d \eta^{2},
$$

where the conformal factor is $\Omega=\tau$, combined with the change from polar Milne coordinates $x^{\mu}=(\tau, r, \phi, \eta)$ to de Sitter coordinates $\hat{x}^{\mu}=(\rho, \theta, \phi, \eta)$ using

$$
\begin{aligned}
\sinh \rho(\tau, r) & =-\frac{1-(q \tau)^{2}+(q r)^{2}}{2 q \tau}, \\
\tan \theta(\tau, r) & =\frac{2 q r}{1+(q \tau)^{2}-(q r)^{2}} .
\end{aligned}
$$

The resulting rescaled line element of the de Sitter spacetime reads

$$
d \hat{s}^{2}=-d \rho^{2}+\cosh ^{2} \rho\left(d \theta^{2}+\sin ^{2} \theta d \phi^{2}\right)+d \eta^{2},
$$

with the metric $\hat{g}_{\mu \nu}=\operatorname{diag}\left(-1, \cosh ^{2} \rho, \cosh ^{2} \rho \sin ^{2} \theta, 1\right)$ (hereafter all quantities defined in the de Sitter space will be denoted with a hat). Clearly, based on Eq. (33), we observe that the Weyl rescaling (30) combined with passing to the standard global coordinates on $d S_{3}$ (31)-(32) promotes the $\left[S O(3)_{q}\right]$ conformal isometry to a manifest isometry $[S O(3)]$ in $(\theta, \phi)$.
In general, for a system to respect conformal symmetry, its dynamics should be invariant under Weyl rescaling [93,94,106-108]. It implies that the $(m, n)$-type tensors [including scalars with $(m, n)=(0,0)$ ] transform homogeneously, namely,

$$
A_{\nu_{1} \ldots \nu_{n}}^{\mu_{1} \ldots \mu_{m}}(x) \rightarrow \Omega^{\Delta_{A}} A_{\nu_{1} \ldots \nu_{n}}^{\mu_{1} \ldots \mu_{m}}(x),
$$

where $\Omega \equiv e^{-\varphi(x)}$ with $\varphi(x)$ being function of spacetime coordinates and $\Delta_{A}=[A]+m-n$ is the conformal weight of the quantity $A$, where $[A]$ is its mass dimension, and $m$ and $n$ are the numbers of contravariant and covariant indices, respectively. For instance, the metric tensor $g_{\mu \nu}$ is a rank-two $(0,2)$ dimensionless tensor, $\left[g_{\mu \nu}\right]=0$, for which one finds $\Delta_{g_{\mu \nu}}=-2$. Hence, $g_{\mu \nu}$ transforms under Weyl rescaling as follows $[94,106]$ :

$$
g_{\mu \nu} \rightarrow \Omega^{-2} g_{\mu \nu} .
$$

Using rules for general coordinate transformations of tensors and Eq. (35) the relation between the $R^{3} \otimes R$ metric and the $d S_{3} \otimes R$ metric is

$$
\hat{g}_{\mu \nu}=\frac{1}{\tau^{2}} \frac{\partial x^{\alpha}}{\partial \hat{x}^{\mu}} \frac{\partial x^{\beta}}{\partial \hat{x}^{\nu}} g_{\alpha \beta} .
$$

Using the information that $\Delta_{g_{\mu \nu}}=-2$ and the unit norm constraint $g_{\mu \nu} U^{\mu} U^{\nu}=-1$, one obtains $\Delta_{U^{\mu}}=1$ which results in the transformation rule

$$
\hat{U}_{\nu}=\frac{1}{\tau} \frac{\partial x^{\mu}}{\partial \hat{x}^{\nu}} U_{\mu}
$$

Using Eq. (37) one can show that the four-velocity profile from Eq. (24) in the de Sitter space is static,

$$
\hat{U}^{\mu}=(1,0,0,0),
$$

meaning it is invariant with respect to the Gubser symmetry group, provided the transverse rapidity profile has the form $[93,94]$

$$
\vartheta(\tau, r)=\tanh ^{-1}\left(\frac{2 q \tau q r}{1+(q \tau)^{2}+(q r)^{2}}\right) .
$$

In a similar manner, the remaining basis vectors (24) in the de Sitter space are

$$
\begin{aligned}
\hat{R}^{\mu} & =\left(0,(\cosh \rho)^{-1}, 0,0\right), \\
\hat{\Phi}^{\mu} & =\left(0,0,(\cosh \rho \sin \theta)^{-1}, 0\right), \\
\hat{Z}^{\mu} & =(0,0,0,1) .
\end{aligned}
$$

The metric $\hat{g}^{\mu \nu}$ can be expressed as 


$$
\hat{g}^{\mu \nu}=-\hat{U}^{\mu} \hat{U}^{\nu}+\hat{R}^{\mu} \hat{R}^{\nu}+\hat{\Phi}^{\mu} \hat{\Phi}^{\nu}+\hat{Z}^{\mu} \hat{Z}^{\nu},
$$

while the determinant of $\hat{g}_{\mu \nu}$ is

$$
\hat{g} \equiv \operatorname{det}\left(\hat{g}_{\mu \nu}\right)=-\cosh ^{4} \rho \sin ^{2} \theta .
$$

Using the fact that the energy density equation (7) and pressure equation (8) have the mass dimension $[\mathcal{E}] \equiv[\mathcal{P}]=4$, their conformal weight is $\Delta_{\mathcal{E}}=\Delta_{\mathcal{P}}=4$. In a similar way, for the net baryon density, one has $[\mathcal{N}]=$ 3 and hence $\Delta_{\mathcal{N}}=3$. Since both temperature and baryon chemical potential have the mass dimension $[T]=[\mu]=1$, their conformal weight is $\Delta_{T}=\Delta_{\mu}=1$. Using an analogous technique to that leading to Eq. (37), the transformation rules needed to map the quantities expressed in de Sitter coordinates back to the polar Milne coordinates can be written as

$$
\begin{array}{rlrl}
U_{\mu}(\tau, r) & =\tau \frac{\partial \hat{x}^{\nu}}{\partial x^{\mu}} \hat{U}_{\nu}(\rho), \\
\mathcal{E}(\tau, r) & =\frac{\hat{\mathcal{E}}(\rho)}{\tau^{4}}, & \mathcal{P}(\tau, r)=\frac{\hat{\mathcal{P}}(\rho)}{\tau^{4}}, & \mathcal{N}(\tau, r)=\frac{\hat{\mathcal{N}}(\rho)}{\tau^{3}} \\
T(\tau, r) & =\frac{\hat{T}(\rho)}{\tau}, & \mu(\tau, r) & =\frac{\hat{\mu}(\rho)}{\tau} .
\end{array}
$$

In Eq. (13), $x^{\beta}$ have mass dimension $\left[x^{\beta}\right]=-1$ with one contravariant index, hence $\Delta_{x^{\beta}}=0$, and using the information about the conformal weight of $\mathcal{E}$ and $U^{\alpha}$ and Eq. (6), one can find that the stress energy tensor should have the conformal weight $\Delta_{T^{\alpha \beta}}=6$. As a result one observes that the conformal weight of the spin tensor is $\Delta_{S^{\alpha \beta \gamma}}=6$, since each term in Eq. (13) should have the same conformal weight. Hence the GLW spin tensor in Eq. (15) [105], as well as canonical spin tensor [109], and the HilgevoordWouthuysen (HW) spin tensor [110,111] should all have the same conformal weight. For instance, for a free Dirac field the canonical spin tensor $S_{\mathrm{C}}^{\alpha, \beta \gamma}$, the GLW spin tensor $S_{\mathrm{GLW}}^{\alpha, \beta \gamma}$, and the HW spin tensor $S_{\mathrm{HW}}^{\alpha, \beta \gamma}$ are defined as $[110,111]$

$$
\begin{gathered}
S_{\mathrm{C}}^{\alpha, \beta \gamma}=\frac{i}{8} \bar{\psi}\left\{\gamma^{\alpha},\left[\gamma^{\beta}, \gamma^{\gamma}\right]\right\} \psi, \\
S_{\mathrm{GLW}}^{\alpha, \beta \gamma}=\frac{i}{4 m}\left(\bar{\psi} \sigma^{\beta \gamma} \stackrel{\leftrightarrow}{\partial}{ }^{\alpha} \psi-\partial_{\rho} \epsilon^{\beta \gamma \alpha \rho} \bar{\psi} \gamma^{5} \psi\right), \\
S_{\mathrm{HW}}^{\alpha, \beta \gamma}=S_{\mathrm{C}}^{\alpha, \beta \gamma}-\frac{1}{4 m}\left(\bar{\psi} \sigma^{\beta \gamma} \sigma^{\alpha \rho} \partial_{\rho} \psi+\partial_{\rho} \bar{\psi} \sigma^{\alpha \rho} \sigma^{\beta \gamma} \psi\right),
\end{gathered}
$$

respectively, with $\sigma^{\mu \nu}=\frac{i}{2}\left[\gamma^{\mu}, \gamma^{\nu}\right]$. Since the conformal weight of both spinor $\psi$ and dual spinor $\bar{\psi} \equiv \psi^{\dagger} \gamma_{0}$ is $\Delta_{\psi}=$ $\Delta_{\bar{\psi}}=\frac{3}{2}$ and the conformal weight of the Dirac gamma matrix is $\Delta_{\gamma^{\mu}}=1[112,113]$, then $\Delta_{S^{\alpha \beta \gamma}}=\Delta_{S_{\mathrm{C}}^{\alpha \beta \gamma}}=\Delta_{S_{\mathrm{HW}}^{\alpha \beta \gamma}}=6$.
Similarly, using the information about the conformal weight of $\mathcal{N}$ in Eq. (2), we find that the conformal weight of the net baryon number current is $\Delta_{N^{\alpha}}=4$.

The conformal weight of the spin polarization tensor $\omega^{\alpha \beta}$ is easily found if one notices the fact that the first term in Eq. (15) should have the same conformal weight as the spin tensor in Eq. (13). In this case for the rank-two dimensionless spin polarization tensor with two contravariant indices, one can find the conformal weight to be $\Delta_{\omega^{\alpha \beta}}=2$. Using this result in Eq. (22), we see that $\Delta_{\kappa^{\alpha}}=1$ and $\Delta_{\omega^{\alpha}}=1$. Here we used the fact that $\epsilon^{\alpha \beta \gamma \delta}$ has zero mass dimension and four contravariant indices, giving $\Delta_{\epsilon^{\alpha \beta \gamma \delta}}=4$. From the information of how $\kappa^{\alpha}, \omega^{\alpha}$ and basis vectors (24) transform under Weyl rescaling and that all the spin polarization components $a_{i}$ and $b_{i}$ are dimensionless scalars, we see that $\Delta_{a_{i}}=$ $\Delta_{b_{i}}=0$. Hence, they are conformally invariant quantities.

Using Eq. (34), we summarize the transformation rules under Weyl rescaling (for four-dimensional spacetime) as follows:

$$
\begin{aligned}
N^{\alpha} & \rightarrow \Omega^{4} N^{\alpha}, \\
T^{\alpha \beta} & \rightarrow \Omega^{6} T^{\alpha \beta}, \\
S^{\alpha \beta \gamma} & \rightarrow \Omega^{6} S^{\alpha \beta \gamma} .
\end{aligned}
$$

Finally, we note that the similar results may be found for the quantities with covariant indices. In particular, one may find that $\Delta_{T_{\alpha \beta}}=2$. The latter result is in agreement with the fact that raising (lowering) the Lorentz index with the metric tensor changes the conformal measure by a factor of $2(-2)$.

\section{CONFORMAL INVARIANCE OF CONSERVATION EQUATIONS}

Considering four-dimensional conformal fluid dynamics, we aim at finding the conformal transformation of the conservation equations for net the baryon number, energy and linear momentum, and spin, whose coordinates are, in general, expressed as

$$
\begin{gathered}
d_{\alpha} N^{\alpha}(x)=\partial_{\alpha} N^{\alpha}+\Gamma_{\alpha \beta}^{\alpha} N^{\beta}=0 \\
d_{\alpha} T^{\alpha \beta}(x)=\partial_{\alpha} T^{\alpha \beta}+\Gamma_{\alpha \lambda}^{\alpha} T^{\lambda \beta}+\Gamma_{\alpha \lambda}^{\beta} T^{\alpha \lambda}=0 \\
d_{\alpha} S^{\alpha \beta \gamma}(x)=\partial_{\alpha} S^{\alpha \beta \gamma}+\Gamma_{\alpha \lambda}^{\alpha} S^{\lambda \beta \gamma}+\Gamma_{\alpha \lambda}^{\beta} S^{\alpha \lambda \gamma}+\Gamma_{\alpha \lambda}^{\gamma} S^{\alpha \beta \lambda}=0,
\end{gathered}
$$

respectively, where $\Gamma_{\alpha \lambda}^{\beta}$ are Christoffel symbols defined in Eq. (A8).

To find the conformal transformations of the conservation laws, (49), (50), and (51), we need to know the conformal transformation of the Christoffel symbols, which is given as $[108,114,115]$ 


$$
\Gamma_{\lambda \alpha}^{\beta}=\hat{\Gamma}_{\lambda \alpha}^{\beta}+\delta_{\lambda}^{\beta} \partial_{\alpha} \varphi+\delta_{\alpha}^{\beta} \partial_{\lambda} \varphi-\hat{g}_{\lambda \alpha} \hat{g}^{\beta \sigma} \partial_{\sigma} \varphi,
$$

where $\delta_{\lambda}^{\beta}$ is the Kronecker delta function and $\varphi$ is a function of spacetime coordinates defined through Eq. (34) [for the derivation of Eq. (52) we refer the reader to Appendix C].

One can show that for the four-dimensional spacetime, the conservation law for the net baryon number (49) is already conformal-frame independent [93,94,106-108], i.e., the net baryon number is conserved in both Minkowski and de Sitter spacetimes. In this case, since the conformal weight of the net baryon number is $\Delta_{N^{\alpha}}=4$, one can write

$$
d_{\alpha} N^{\alpha}=\Omega^{4} \hat{d}_{\alpha} \hat{N}^{\alpha} .
$$

Using Eq. (47) and Eq. (52) in Eq. (50), we find that the conservation of energy and linear momentum transforms as $[107,108,114]$

$$
d_{\alpha} T^{\alpha \beta}=\Omega^{6}\left[\hat{d}_{\alpha} \hat{T}^{\alpha \beta}-\hat{T}_{\lambda}^{\lambda} \hat{g}^{\beta \delta} \partial_{\delta} \varphi\right] .
$$

Hence, we observe that $\hat{T}^{\alpha \beta}$ needs to be traceless in order to be conserved in de Sitter spacetime. Therefore, the breaking of conformal invariance is characterized only by the trace of the energy-momentum tensor ${ }^{1}$ [116-119].

Furthermore, using Eq. (48) and Eq. (52) in Eq. (51), the conformal transformation of the conservation law for spin takes the form

$$
d_{\alpha} S^{\alpha \beta \gamma}=\Omega^{6}\left[\hat{d}_{\alpha} \hat{S}^{\alpha \beta \gamma}-\left(\hat{S}_{\lambda}^{\lambda \gamma} \hat{g}^{\beta \sigma}+\hat{S}^{\alpha \beta}{ }_{\alpha} \hat{g}^{\sigma \gamma}\right) \partial_{\sigma} \varphi\right] .
$$

From Eq. (55) we find that the conformal invariance of the spin conservation law requires the spin tensor to satisfy the condition $\hat{S}_{\alpha}{ }^{\alpha \beta}=0$. It is straightforward to show that the GLW (15), (44) and HW (45) definitions do not satisfy this condition, and hence, explicitly break the conformal invariance of Eq. (55). The consequences of this issue will be discussed in the next section.

\section{DYNAMICS IN DE SITTER COORDINATES}

In this section we explore the dynamics of the spin polarization on top of the conformal Gubser-expanding hydrodynamic background in the de Sitter spacetime. Our final results are obtained by transforming back to the Minkowski spacetime using Eq. (42).

\section{A. Conformal symmetry breaking and the equation of state}

In Sec. VI we have shown that Eqs. (1) and (5) are conformally invariant, i.e., they transform homogeneously under the Weyl transformation provided the constitutive relations do so and the energy-momentum tensor is

\footnotetext{
${ }^{1}$ Note that the latter holds regardless of the symmetry of the energy-momentum tensor.
}

traceless. As the presence of finite masses breaks the tracelessness condition [(in our case $\hat{T}_{\lambda}^{\lambda}=3 \hat{\mathcal{P}}-\hat{\mathcal{E}} \approx$ $\mathcal{O}\left(m^{2}\right)$ ], in order to respect Gubser symmetry and keep the four-velocity pattern invariant, the energy density (7), pressure (8), and net baryon density (3) have to be treated in the massless limit, meaning

$$
\begin{aligned}
& \hat{\mathcal{E}}=24 k \cosh \alpha \hat{T}^{4}, \\
& \hat{\mathcal{P}}=8 k \cosh \alpha \hat{T}^{4}, \\
& \hat{\mathcal{N}}=8 k \sinh \alpha \hat{T}^{3},
\end{aligned}
$$

respectively. Obviously, in this case one has $\hat{\mathcal{E}}=3 \hat{\mathcal{P}}$.

On the other hand, for the conservation of spin to respect conformal invariance, it is sufficient that the spin tensor in Eq. (55) is totally antisymmetric. ${ }^{2}$ As noticed in Sec. VI, the GLW form (15) of the spin tensor considered in this work does not satisfy this requirement. In general, if Eqs. (1), (5), and (14) were fully coupled, this would lead to the breaking of the Gubser symmetry and, in consequence, of the flow invariance. However, in the particular case studied herein, this is not the case since the spin dynamics given by Eq. (14) is treated only perturbatively [22], and hence decouples from the background hydrodynamic fields. This allows for a separate solution of background equations of motion (1) and (5) and the subsequent solution of the spin part (14). As there is no backreaction to the background from the evolution of spin dynamics [the polarization tensor does not enter Eqs. (1) and (5)], the conformal-breaking dynamics of spin polarization does not spoil the flow invariance (a similar issue was encountered also in other recent works, see, for instance, Ref. [120]). At this point, it is important to mention that, despite this issue, we are still allowed to investigate the spin dynamics in the de Sitter coordinates, which we will do for the sake of convenience. Using the same arguments as given above, we will also keep finite masses in expressions for $\mathcal{A}, \mathcal{B}$, and $\mathcal{C}$ defining the GLW spin tensor in Eqs. (15) and (16).

\section{B. Perfect-fluid background}

Using Eq. (2) in Eq. (1) the conservation law for charge in the de Sitter coordinates can be written as

$$
\hat{U}^{\alpha} \partial_{\alpha} \hat{\mathcal{N}}+\hat{\mathcal{N}} \partial_{\alpha} \hat{U}^{\alpha}+\hat{\mathcal{N}} \hat{U}^{\alpha} \frac{\partial_{\alpha} \sqrt{-\hat{g}}}{\sqrt{-\hat{g}}}=0,
$$

where $\hat{g}$ is the determinant of the de Sitter metric (41) and we employed definitions of the covariant derivative from

\footnotetext{
${ }^{2}$ Note that this is not the case for the GLW and HW definitions whose additional terms $\sim 1 / m$ make them ill-defined in the massless limit as well as break the conformal invariance of the spin conservation.
} 
Appendix A. Identifying expansion scalar as $\hat{\theta} \equiv 2 \tanh \rho$, one may rewrite Eq. (59) in the following form:

$$
\partial_{\rho} \hat{\mathcal{N}}+\hat{\mathcal{N}} \hat{\theta}=0
$$

In a similar way, transforming Eq. (5) to $d S_{3} \otimes R$, using Eq. (6), and contracting it with $\hat{U}_{\beta}$, we get the conservation law for energy as follows:

$$
\partial_{\rho} \hat{\mathcal{E}}+(\hat{\mathcal{E}}+\hat{\mathcal{P}}) \hat{\theta}=0 .
$$

One may check that all other projections of Eq. (5) are satisfied identically.

The solutions of Eq. (60) and Eq. (61) can be found analytically giving $[93,94]$,

$$
\begin{aligned}
& \hat{\mathcal{E}}=\hat{\mathcal{E}}_{0}\left(\frac{\cosh \rho_{0}}{\cosh \rho}\right)^{8 / 3}, \\
& \hat{\mathcal{N}}=\hat{\mathcal{N}}_{0}\left(\frac{\cosh \rho_{0}}{\cosh \rho}\right)^{2},
\end{aligned}
$$

respectively, where $\hat{\mathcal{E}}_{0} \equiv \hat{\mathcal{E}}\left(\rho_{0}\right)$ and $\hat{\mathcal{N}}_{0} \equiv \hat{\mathcal{N}}\left(\rho_{0}\right)$ are integration constants and $\rho_{0}$ is the initial de Sitter time. Using Eqs. (56)-(58), the corresponding solutions for temperature and baryon chemical potential in de Sitter space are

$$
\begin{aligned}
& \hat{T}=\hat{T}_{0}\left(\frac{\cosh \rho_{0}}{\cosh \rho}\right)^{2 / 3}, \\
& \hat{\mu}=\hat{\mu}_{0}\left(\frac{\cosh \rho_{0}}{\cosh \rho}\right)^{2 / 3},
\end{aligned}
$$

where, again, $\hat{T}_{0} \equiv \hat{T}\left(\rho_{0}\right)$ and $\hat{\mu}_{0} \equiv \hat{\mu}\left(\rho_{0}\right)$ are constants of integration. Based on the above solutions, we observe that $\hat{\alpha}$ is a $\rho$ independent quantity. Moreover, as shown in $[93,94]$ one can see that the Gubser symmetry restricts the dynamics of the system in such a way that it depends only on the de Sitter time $\rho$.

The spacetime evolution of temperature as given by Eq. (64) and flow-vector components $\left(U^{\tau}, U^{r}\right) /$ $\sqrt{\left(U^{\tau}\right)^{2}+\left(U^{r}\right)^{2}}$ is shown in Fig. 1. For setting initial temperature parameters we followed Ref. [121] where it

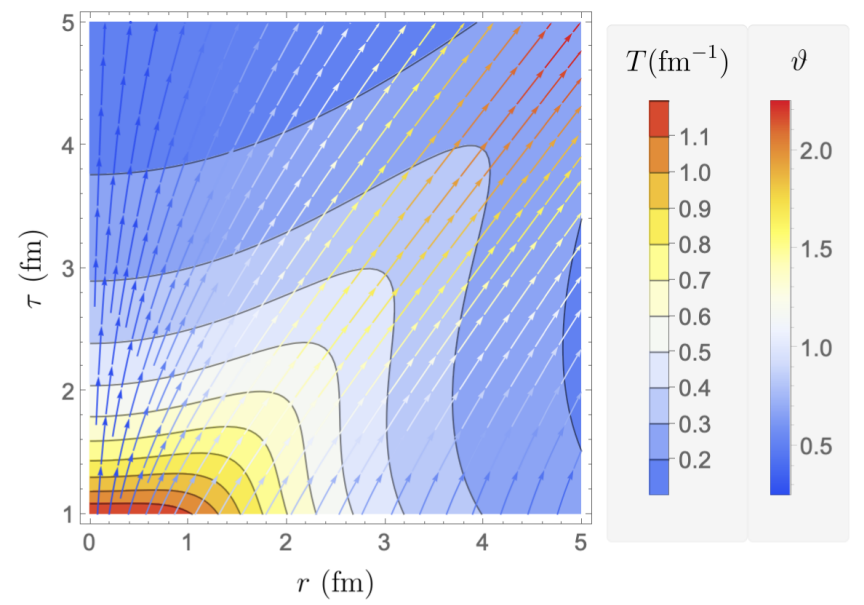

FIG. 1. The spacetime dependence of temperature (contours) and flow-vector components $\left(U^{\tau}, U^{r}\right) / \sqrt{\left(U^{\tau}\right)^{2}+\left(U^{r}\right)^{2}}$ (stream lines: the coloring of arrows is given by the rapidity $\vartheta$ ); see the bar legends for the scalings.

was assumed that $\hat{T}_{0} \equiv \hat{T}\left(\rho_{0}\right)=1.2$ at $\rho_{0}=0$ so that choosing $q=1 \mathrm{fm}^{-1}$ results in $T\left(\tau_{0}=1 \mathrm{fm}, r=0\right)=$ $1.2 \mathrm{fm}^{-1}$. One can observe that the temperature and flow profiles are strongly correlated.

\section{Spin evolution}

In this section we derive the spin evolution equations which we use to study spin dynamics in the Gubser-flowing perfect-fluid background presented in the previous section. Herein, we assume that the spin polarization components, apart from $\rho$ dependence, also exhibit $\theta$ dependence. Hence, following the findings in Sec. VI, we assume that, in general, spin equations of motion break the conformal invariance. In such a case $\rho$ and $\theta$ coordinates in the de Sitter space serve just as an alternative parametrization of the directions $(\tau, r)$ in polar Milne coordinates. By substituting Eq. (15) with Eq. (16), Eq. (28) and Eq. (29) in Eq. (14), employing Eqs. (64)-(65), and subsequently projecting the resulting tensorial equation on $\hat{U}_{\beta} \hat{R}_{\gamma}, \hat{U}_{\beta} \hat{\Phi}_{\gamma}, \hat{U}_{\beta} \hat{Z}_{\gamma}, \hat{\Phi}_{\beta} \hat{Z}_{\gamma}, \hat{R}_{\beta} \hat{Z}_{\gamma}$, and $\hat{R}_{\beta} \hat{\Phi}_{\gamma}$ we obtain the following equations of motion for the spin polarization components:

$$
\begin{gathered}
\hat{\mathcal{B}} \dot{\hat{a}}_{R}=-\hat{a}_{R}\left[\dot{\hat{\mathcal{B}}}+\frac{5}{2} \hat{\mathcal{B}} \tanh \rho\right], \\
\hat{\mathcal{B}} \dot{\hat{a}}_{\Phi}+\frac{\hat{\mathcal{B}}}{2} \cosh \rho \sin \theta \hat{b}_{Z}=-\hat{a}_{\Phi}\left[\dot{\hat{\mathcal{B}}}+\frac{5}{2} \hat{\mathcal{B}} \tanh \rho\right]-\hat{b}_{Z} \frac{\hat{\mathcal{B}}}{2} \cosh \rho \cos \theta, \\
\hat{\mathcal{B}} \dot{\hat{a}}_{Z}-\frac{\hat{\mathcal{B}}}{2} \cosh \rho \sin \theta \hat{b}_{\Phi}=-\hat{a}_{Z}[\dot{\hat{\mathcal{B}}}+3 \hat{\mathcal{B}} \tanh \rho]+\hat{b}_{\Phi} \hat{\mathcal{B}} \cosh \rho \cos \theta \\
(\hat{\mathcal{B}}-\hat{\mathcal{C}}) \dot{\hat{b}}_{R}=-\hat{b}_{R}\left[(\dot{\hat{\mathcal{B}}}-\dot{\hat{\mathcal{C}}})+\left(\frac{9 \hat{\mathcal{B}}}{2}-4 \hat{\mathcal{C}}\right) \tanh \rho\right],
\end{gathered}
$$




$$
\begin{gathered}
(\hat{\mathcal{B}}-\hat{\mathcal{C}}) \dot{\hat{b}}_{\Phi}-\frac{\hat{\mathcal{B}}}{2 \sin \theta}(\operatorname{sech} \rho)^{3} \hat{a}_{Z}=-\hat{b}_{\Phi}\left[(\dot{\hat{\mathcal{B}}}-\dot{\hat{\mathcal{C}}})+\left(\frac{9 \hat{\mathcal{B}}}{2}-4 \hat{\mathcal{C}}\right) \tanh \rho\right], \\
(\hat{\mathcal{B}}-\hat{\mathcal{C}}) \dot{\hat{b}}_{Z}+\frac{\hat{\mathcal{B}}}{2 \sin \theta}(\operatorname{sech} \rho)^{3} \hat{a}_{\Phi}=-\hat{b}_{Z}[(\dot{\hat{\mathcal{B}}}-\dot{\hat{\mathcal{C}}})+(5 \hat{\mathcal{B}}-4 \hat{\mathcal{C}}) \tanh \rho]-\frac{\hat{\mathcal{B}}}{2 \sin \theta} \cot \theta(\operatorname{sech} \rho)^{3} \hat{a}_{\Phi},
\end{gathered}
$$

respectively, where $\left({ }^{\circ}\right) \equiv \partial_{\rho},\left(^{\circ}\right) \equiv \partial_{\theta}$, and $\hat{\mathcal{B}}$ and $\hat{\mathcal{C}}$ are defined in Eq. (18) and Eq. (19). In the latter expressions, unlike in the case of hydrodynamic background, we use full expressions for energy density (7) and pressure (8) expressed in terms of finite particle masses.

\section{Massless limit}

From Eqs. (66)-(71) we observe that, unlike in the case of Bjorken expansion [22], only spin polarization components along $R^{\mu}$ evolve independently when expressed in de Sitter coordinates. On the other hand $\hat{a}_{\Phi}\left(\hat{b}_{\Phi}\right)$ and $\hat{b}_{Z}\left(\hat{a}_{Z}\right)$, respectively, are coupled to each other. The coupling between the components emerge due to the conformal symmetry breaking, and manifests itself through the $\theta$ dependence of the latter.

In the massless case, the solutions to Eqs. (66) and (69) may be found analytically giving

$$
\begin{aligned}
& \hat{a}_{R}=\hat{a}_{R}^{0}\left(\frac{\cosh \rho}{\cosh \rho_{0}}\right)^{5 / 6}, \\
& \hat{b}_{R}=\hat{b}_{R}^{0}\left(\frac{\cosh \rho_{0}}{\cosh \rho}\right)^{7 / 6},
\end{aligned}
$$

where $\hat{a}_{R}^{0} \equiv \hat{a}_{R}\left(\rho_{0}\right)$ and $\hat{b}_{R}^{0} \equiv \hat{b}_{R}\left(\rho_{0}\right)$. The characteristic concave dependence of $\hat{b}_{R}$ on de Sitter time is qualitatively similar to that of temperature and baryon chemical potential (in the case of $\hat{a}_{R}$ one deals with the convex function of $\rho$ ).

The dynamics of $\hat{b}_{\Phi}$ and $\hat{a}_{Z}$ components following from Eqs. (68) and (70) is more complicated, however, it shows certain characteristic features. In particular, if $\hat{b}_{\Phi}$ is initially negligible, the $\hat{a}_{Z}$ component is approximately $\theta$ independent, yielding

$$
\left.\hat{a}_{Z}\right|_{\hat{b}_{\Phi}=0}=\hat{a}_{Z}^{0}\left(\frac{\cosh \rho}{\cosh \rho_{0}}\right)^{1 / 3},
$$

with $\hat{a}_{Z}^{0} \equiv \hat{a}_{Z}\left(\rho_{0}\right)$, i.e., giving $\hat{a}_{Z}(\rho) \sim 1 / \sqrt{\hat{T}(\rho)}$.

In the general case where $\hat{b}_{\Phi} \neq 0$, one may use the fact that $\hat{a}_{Z}$ is a slowly varying function of $\theta$ and the second term on the left-hand side of Eq. (70) may be neglected. In this case, the solution to the $\hat{b}_{\Phi}$ component has the approximate form

$$
\hat{b}_{\Phi} \approx \hat{b}_{\Phi}^{0}\left(\frac{\cosh \rho_{0}}{\cosh \rho}\right)^{7 / 6}
$$

where $\hat{b}_{\Phi}^{0} \equiv \hat{b}_{\Phi}\left(\rho_{0}\right)$. Using the exact numerical solutions, one can show that, indeed, $\hat{b}_{\Phi}$ is a weakly dependent function of $\theta$ and hence approximately proportional to $\hat{b}_{R}$.

In the case of the remaining $\hat{a}_{\Phi}$ and $\hat{b}_{Z}$ components, simple solutions cannot be found and, in general, Eqs. (67)
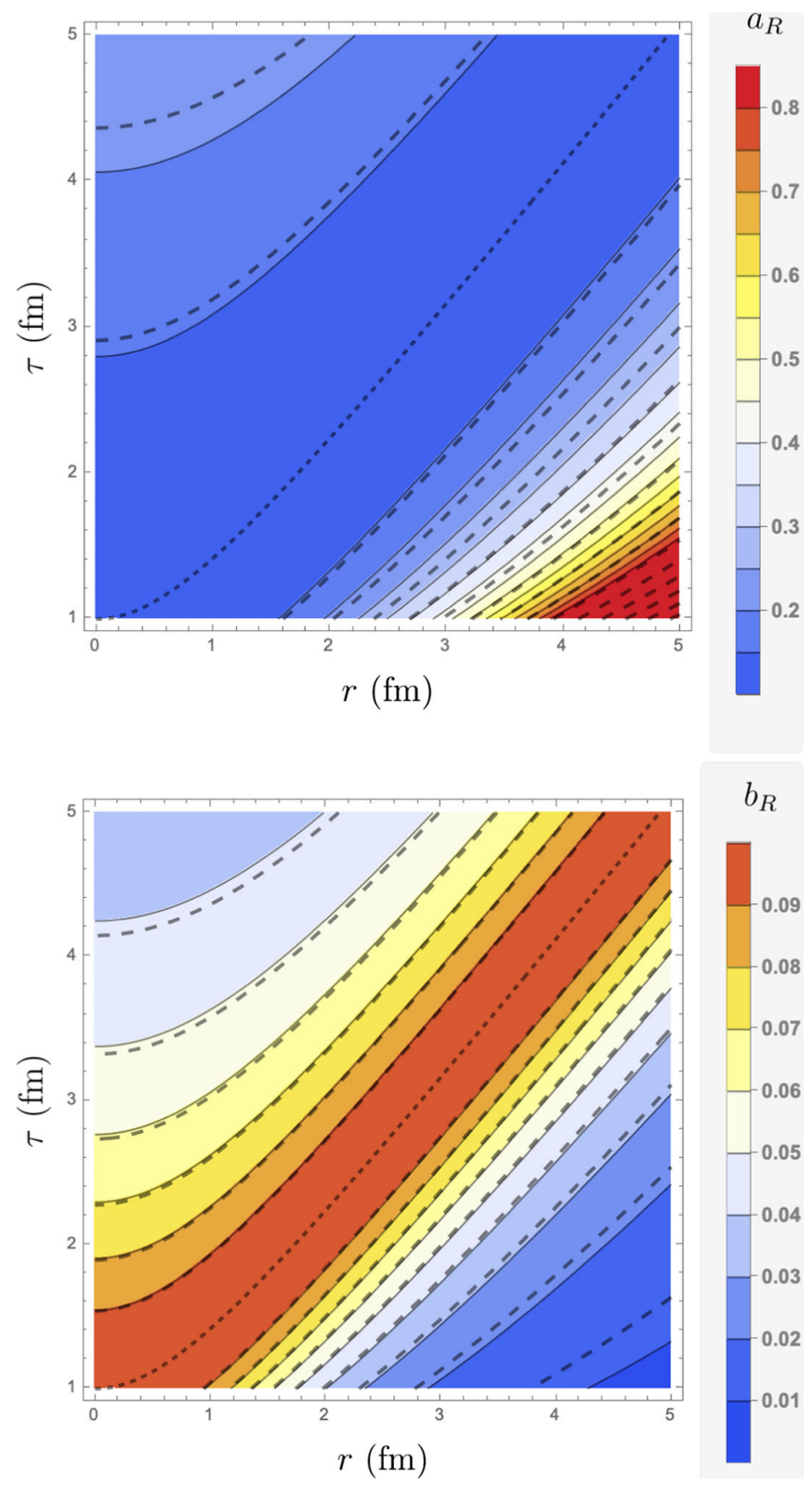

FIG. 2. Numerical solutions for $a_{R}$ and $b_{R}$ components of the spin polarization tensor as functions of proper time $\tau$ and radial distance $r$. 
and (71) have to be solved numerically. However, due to the specific structure of Eqs. (67) and (71) one may find some special solutions by requiring the $\theta$ terms to vanish, thus making the $\hat{a}_{\Phi}$ and $\hat{b}_{Z}$ independent of each other. The latter takes place for the following solutions:

$$
\begin{aligned}
& \hat{a}_{\Phi} \approx \hat{a}_{\Phi}^{0}\left(\frac{\cosh \rho}{\cosh \rho_{0}}\right)^{5 / 6} \csc \theta \\
& \hat{b}_{Z} \approx \hat{b}_{Z}^{0}\left(\frac{\cosh \rho_{0}}{\cosh \rho}\right)^{5 / 3} \csc \theta
\end{aligned}
$$

where, again, $\hat{a}_{\Phi}^{0} \equiv \hat{a}_{\Phi}\left(\rho_{0}\right)$ and $\hat{b}_{Z}^{0} \equiv \hat{b}_{Z}\left(\rho_{0}\right)$, and hence $\hat{a}_{\Phi} \sim \hat{a}_{R}$. Finally, we observe that, in the cases discussed
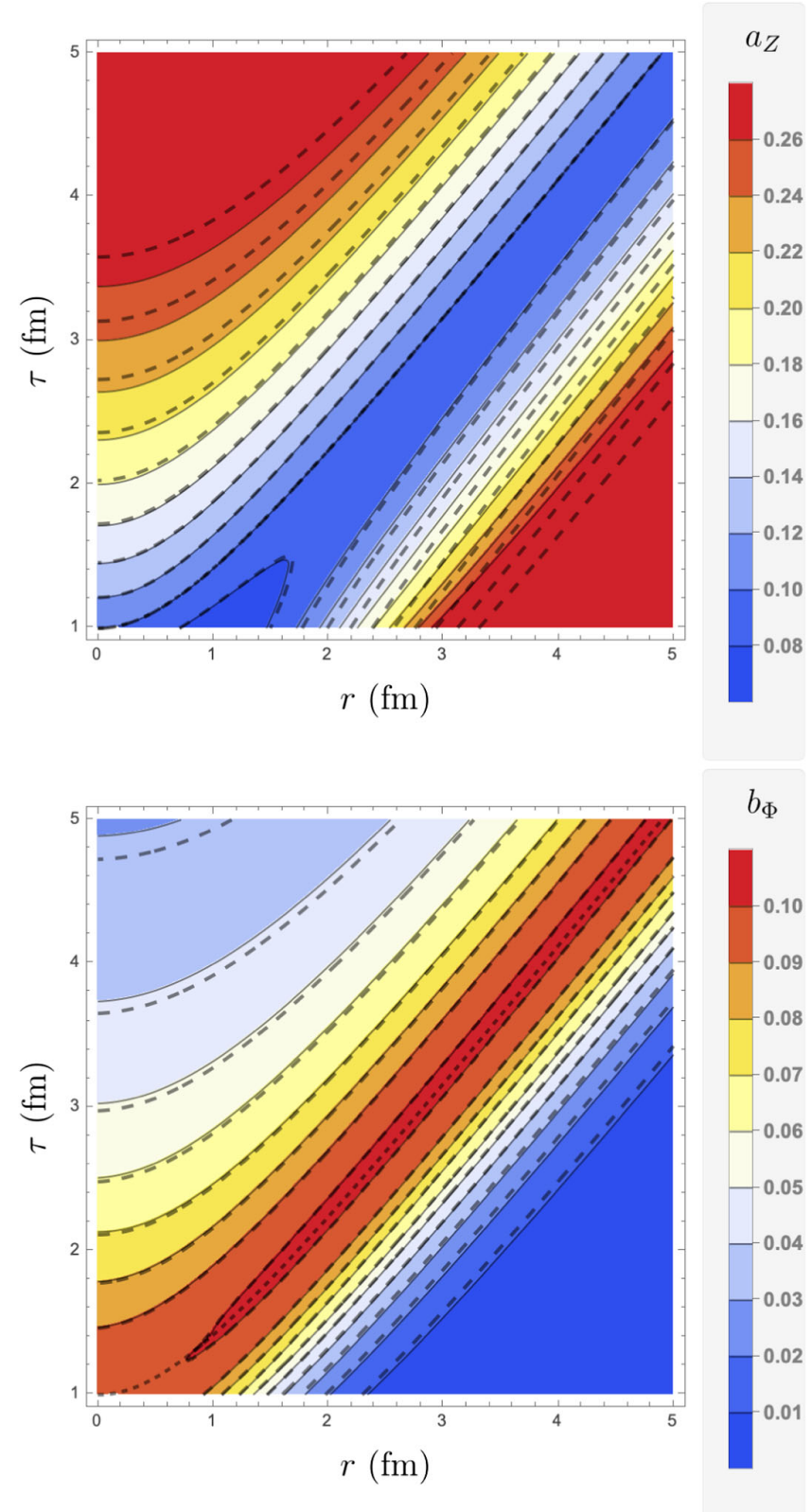

FIG. 3. Same as Fig. 2 but for $a_{Z}$ and $b_{\Phi}$ components. above all components exhibit the universal dependence of the type $(\cosh \rho)^{c}$, with $c$ being positive constant for $a_{i}$ and negative for $b_{i}$.

\section{Numerical results}

Let us turn to some numerical solutions of the spin equations of motion. In Fig. 2 we present the exact numerical solutions of Eqs. (66) and (69) for $a_{R}$ and $b_{R}$ components as functions of proper time $\tau$ and radial distance $r$. The initial values of the $\hat{a}_{R}$ and $\hat{b}_{R}$ components are chosen in such a way so that $\hat{a}_{R}^{0}=0.1$ and $\hat{b}_{R}^{0}=0.1$, which implies $a_{R}\left(\tau_{0}=1 \mathrm{fm}, r=0\right)=b_{R}\left(\tau_{0}=1 \mathrm{fm}, r=0\right)=0.1$. The mass in the calculations is set to $m=0.5 \hat{T}_{0}$. We find that the dynamics of $a_{R}$ and $b_{R}$ components is qualitatively different with $a_{R}$ having minimum and $b_{R}$ having a maximum at $\rho_{0}$ (the black dotted lines). Moreover, by comparing with the massless case we checked (see dashed lines) that the mass is weakly affecting the polarization dynamics as long as the mass is small, i.e., $z<1$.

In Fig. 3 we present analogous plots to Fig. 2 but for $a_{Z}$ and $b_{\Phi}$ components using the numerical solutions of Eqs. (68) and (70). In this case we initialize the components in a similar way as previously assuming $\hat{a}_{Z}^{0}=0.1$ and $\hat{b}_{\Phi}^{0}=0.1$. We observe a weak $\theta$ dependence of the solutions which is due to mixing of the two components. A comparison to the massless case (dashed lines) confirms a small sensitivity of the solution to the mass of the particles. Moreover, we find that the increase of mass in general increases the values of the spin polarization components within the considered region. Similar conclusions hold for the remaining $a_{\Phi}$ and $b_{Z}$ components.

\section{SUMMARY AND CONCLUSIONS}

In this paper we have used the formalism of hydrodynamics with spin $[22,25]$ to derive the equations of motion for spin polarization in the Gubser-expanding conformal hydrodynamic background [93,94]. Following previous works on this topic we have first solved the perfect-fluid hydrodynamical equations using the Gubser symmetry arguments in the de Sitter coordinates and obtained analytical solutions for hydrodynamic variables; see Eqs. (62)-(65). Subsequently, we have extended the analysis of the properties of the conservation laws with respect to the conformal transformation to the case of the angular momentum conservation. We have found that the latter is conformally invariant provided the spin tensor is completely antisymmetric and the particles are massless. As, in general, the currents in the GLW framework studied here break these requirements, we have solved the spin dynamics in the Gubser-expanding background in the de Sitter coordinates (for the sake of convenience) allowing, however, for both $\rho$ and $\theta$ dependence, of the spin polarization components. As there is no backreaction of the latter on the background, we performed this analysis in 
the general massive case. The solutions obtained in this way are mapped back to the Milne coordinates, giving us the complete spatiotemporal evolution for the system with boost invariance and cylindrical symmetry. In the massless limit we have found certain special solutions which, to a large extent, exhibit power-law type dependence on temperature. We have found that, unlike in the Bjorken case, only radial components behave independently.

The framework used herein might be used, for instance, for the description of head-on collisions of initially polarized particles/ions at high energies [122,123], describing the mixing between polarization components along beam and in the azimuthal direction. Moreover, the formalism worked out here can be used for cross-checking the numerical simulations within the full $3+1 \mathrm{D}$ geometry framework, which is now being developed.

\section{ACKNOWLEDGMENTS}

We would like to thank Sayantani Bhattacharyya, Wojciech Florkowski, Masoud Shokri, David Sénéchal, and Yifan Wang for inspiring discussions and clarifications. R. S. would like to acknowledge the kind hospitality during the school "Frontiers in Nuclear and Hadronic Physics 2020" at The Galileo Galilei Institute for Theoretical Physics in Florence, Italy where this work was initiated and also thank the Yukawa Institute for Theoretical Physics at Kyoto University where discussions during the YITP workshop YITP-W-20-03 on "Strings and Fields 2020" were useful to complete this work. This research was supported in part by the Polish National Science Center Grants No. 2016/23/B/ ST2/00717 and No. 2018/30/E/ST2/00432.

\section{APPENDIX A: THE COVARIANT DERIVATIVE}

In a more general spacetime, the partial derivative operator is not a good tensorial operator; therefore, we would need the covariant derivative operator which is an extension of the partial derivative to arbitrary manifolds, and which reduces to a partial derivative operator in flat spacetime in Cartesian coordinates. Its action on an arbitrary scalar $V$, rank-one $V^{\mu}$, and rank-two $V^{\mu \nu}$ tensors is

$$
\begin{gathered}
d_{\mu} V=\partial_{\mu} V, \\
d_{\mu} V_{\nu}=\partial_{\mu} V_{\nu}-\Gamma_{\mu \nu}^{\sigma} V_{\sigma}, \\
d_{\lambda} V_{\mu \nu}=\partial_{\lambda} V_{\mu \nu}-\Gamma_{\lambda \mu}^{\sigma} V_{\sigma \nu}-\Gamma_{\lambda \nu}^{\sigma} V_{\mu \sigma}, \\
d_{\mu} V^{\nu}=\partial_{\mu} V^{\nu}+\Gamma_{\mu \sigma}^{\nu} V^{\sigma}, \\
d_{\mu} V^{\mu}=\partial_{\mu} V^{\mu}+\Gamma_{\mu \sigma}^{\mu} V^{\sigma}, \\
d_{\mu} V^{\mu \nu}=\partial_{\mu} V^{\mu \nu}+\Gamma_{\mu \sigma}^{\mu} V^{\sigma \nu}+\Gamma_{\mu \sigma}^{\nu} V^{\mu \sigma}, \\
d_{\lambda} V^{\mu \nu}=\partial_{\lambda} V^{\mu \nu}+\Gamma_{\lambda \sigma}^{\mu} V^{\sigma \nu}+\Gamma_{\lambda \sigma}^{\nu} V^{\mu \sigma},
\end{gathered}
$$

where $d$ denotes covariant derivative, $\partial$ is partial derivative, $g=\operatorname{det} g_{\mu \nu}$ and $\Gamma_{\mu \lambda}^{\nu}$ are Christoffel symbols which are expressed as

$$
\Gamma_{\mu \lambda}^{\nu} \equiv \Gamma_{\lambda \mu}^{\nu}=\frac{1}{2} g^{\nu \sigma}\left(\partial_{\mu} g_{\sigma \lambda}+\partial_{\lambda} g_{\sigma \mu}-\partial_{\sigma} g_{\mu \lambda}\right)
$$

\section{APPENDIX B: CHRISTOFFEL SYMBOLS IN DE SITTER COORDINATES}

Starting from the definition of the Christoffel symbol (A8) and using the de Sitter spacetime metric (33), the following Christoffel symbols, which do not vanish, can be obtained:

$$
\begin{gathered}
\Gamma_{\theta \theta}^{\rho}=\sinh \rho \cosh \rho, \\
\Gamma_{\phi \phi}^{\rho}=\sin ^{2} \theta \sinh \rho \cosh \rho, \\
\Gamma_{\rho \theta}^{\theta}=\Gamma_{\theta \rho}^{\theta}=\tanh \rho, \\
\Gamma_{\phi \phi}^{\theta}=-\sin \theta \cos \theta, \\
\Gamma_{\rho \phi}^{\phi}=\Gamma_{\phi \rho}^{\phi}=\tanh \rho, \\
\Gamma_{\theta \phi}^{\phi}=\Gamma_{\phi \theta}^{\phi}=\cot \theta .
\end{gathered}
$$

\section{APPENDIX C: CONFORMAL TRANSFORMATION OF CHRISTOFFEL SYMBOLS}

In the de Sitter spacetime metric, the Christoffel symbol equation (A8) is written as below where we use the transformation law of the metric tensor equation (35) to obtain the conformal transformation of the Christoffel symbol

$$
\begin{aligned}
\hat{\Gamma}_{\mu \lambda}^{\nu}= & \frac{1}{2} \hat{g}^{\nu \sigma}\left[\partial_{\mu} \hat{g}_{\sigma \lambda}+\partial_{\lambda} \hat{g}_{\sigma \mu}-\partial_{\sigma} \hat{g}_{\mu \lambda}\right], \\
& \text { using Eq. }(35), \\
\hat{\Gamma}_{\mu \lambda}^{\nu}= & \frac{1}{2} \Omega^{-2} g^{\nu \sigma}\left[\partial_{\mu}\left(\Omega^{2} g_{\sigma \lambda}\right)+\partial_{\lambda}\left(\Omega^{2} g_{\sigma \mu}\right)-\partial_{\sigma}\left(\Omega^{2} g_{\mu \lambda}\right)\right], \\
= & \frac{e^{2 \varphi} g^{\nu \sigma}}{2}\left[\partial_{\mu}\left(e^{-2 \varphi} g_{\sigma \lambda}\right)+\partial_{\lambda}\left(e^{-2 \varphi} g_{\sigma \mu}\right)-\partial_{\sigma}\left(e^{-2 \varphi} g_{\mu \lambda}\right)\right], \\
= & \frac{e^{2 \varphi} g^{\nu \sigma}}{2}\left[2 e^{-\varphi} \partial_{\mu}\left(e^{-\varphi}\right) g_{\sigma \lambda}+e^{-2 \varphi} \partial_{\mu}\left(g_{\sigma \lambda}\right)\right. \\
& +2 e^{-\varphi} \partial_{\lambda}\left(e^{-\varphi}\right) g_{\sigma \mu}+e^{-2 \varphi} \partial_{\lambda}\left(g_{\sigma \mu}\right) \\
& \left.-2 e^{-\varphi} \partial_{\sigma}\left(e^{-\varphi}\right) g_{\mu \lambda}-e^{-2 \varphi} \partial_{\sigma}\left(g_{\mu \lambda}\right)\right], \\
= & \frac{g^{\nu \sigma}}{2}\left[\partial_{\mu}\left(g_{\sigma \lambda}\right)+\partial_{\lambda}\left(g_{\sigma \mu}\right)-\partial_{\sigma}\left(g_{\mu \lambda}\right)\right. \\
& \left.+e^{\varphi}\left(\partial_{\mu}\left(e^{-\varphi}\right) g_{\sigma \lambda}+\partial_{\lambda}\left(e^{-\varphi}\right) g_{\sigma \mu}-\partial_{\sigma}\left(e^{-\varphi}\right) g_{\mu \lambda}\right)\right], \\
= & \Gamma_{\mu \lambda}^{\nu}+e^{\varphi}\left[\delta_{\lambda}^{\nu} \partial_{\mu}\left(e^{-\varphi}\right)+\delta_{\mu}^{\nu} \partial_{\lambda}\left(e^{-\varphi}\right)-\partial_{\sigma}\left(e^{-\varphi}\right) g^{\nu \sigma} g_{\mu \lambda}\right], \\
\Gamma_{\mu \lambda}^{\nu}= & \hat{\Gamma}_{\mu \lambda}^{\nu}+\delta_{\lambda}^{\nu} \partial_{\mu} \varphi+\delta_{\mu}^{\nu} \partial_{\lambda} \varphi-\hat{g}^{\nu \sigma} \hat{g}_{\mu \lambda} \partial_{\sigma} \varphi .
\end{aligned}
$$

Here we note that for $\varphi(x)=$ const., one has $\hat{\Gamma}_{\mu \lambda}^{\nu}=\Gamma_{\mu \lambda}^{\nu}$. 
[1] W. Florkowski, Phenomenology of Ultra-Relativistic Heavy-Ion Collisions (World Scientific, Singapore, 2010), https://cds.cern.ch/record/1321594.

[2] C. Gale, S. Jeon, and B. Schenke, Hydrodynamic modeling of heavy-ion collisions, Int. J. Mod. Phys. A 28, 1340011 (2013).

[3] S. Jeon and U. Heinz, Introduction to hydrodynamics, Int. J. Mod. Phys. E 24, 1530010 (2015).

[4] A. Jaiswal and V. Roy, Relativistic hydrodynamics in heavy-ion collisions: General aspects and recent developments, Adv. High Energy Phys. 2016, 9623034 (2016).

[5] P. Romatschke and U. Romatschke, Relativistic Fluid Dynamics In and Out of Equilibrium, Cambridge Monographs on Mathematical Physics (Cambridge University Press, Cambridge, England, 2019).

[6] W. Florkowski, M. P. Heller, and M. Spalinski, New theories of relativistic hydrodynamics in the LHC era, Rep. Prog. Phys. 81, 046001 (2018).

[7] M. Alqahtani, M. Nopoush, and M. Strickland, Relativistic anisotropic hydrodynamics, Prog. Part. Nucl. Phys. 101, 204 (2018).

[8] J. Berges, M. P. Heller, A. Mazeliauskas, and R. Venugopalan, Thermalization in QCD: Theoretical approaches, phenomenological applications, and interdisciplinary connections, arXiv:2005.12299.

[9] B. I. Abelev et al. (STAR Collaboration), Global polarization measurement in $\mathrm{Au}+\mathrm{Au}$ collisions, Phys. Rev. $\mathrm{C}$ 76, 024915 (2007); , Erratum, Phys. Rev. C 95, 039906 (2017).

[10] L. Adamczyk et al. (STAR Collaboration), Global $\Lambda$ hyperon polarization in nuclear collisions: Evidence for the most vortical fluid, Nature (London) 548, 62 (2017).

[11] J. Adam et al. (STAR Collaboration), Global polarization of $\Lambda$ hyperons in $\mathrm{Au}+\mathrm{Au}$ collisions at $\sqrt{s_{N N}}=200 \mathrm{GeV}$, Phys. Rev. C 98, 014910 (2018).

[12] J. Adam et al. (STAR Collaboration), Polarization of $\Lambda(\bar{\Lambda})$ Hyperons Along the Beam Direction in $\mathrm{Au}+\mathrm{Au}$ Collisions at $\sqrt{s_{N N}}=200 \mathrm{GeV}$, Phys. Rev. Lett. 123, 132301 (2019).

[13] S. Acharya et al. (ALICE Collaboration), Measurement of Spin-Orbital Angular Momentum Interactions in Relativistic Heavy-Ion Collisions, Phys. Rev. Lett. 125, 012301 (2020).

[14] F. Kornas et al. (HADES Collaboration), Lambda polarization in $\mathrm{Au}+\mathrm{Au}$ collisions at $\sqrt{s_{N N}}=2.4 \mathrm{GeV}$ measured with HADES, in Proceedings at the Strange Quark Matter, Bari, Italy (Springer, Cham, 2019), https://doi.org/ 10.1007/978-3-030-53448-6_68.

[15] S. Acharya et al. (ALICE Collaboration), Global polarization of $\Lambda \bar{\Lambda}$ hyperons in $\mathrm{Pb}-\mathrm{Pb}$ collisions at $\sqrt{s_{N N}}=2.76$ and 5.02 TeV, Phys. Rev. C 101, 044611 (2020).

[16] W. Florkowski, B. Friman, A. Jaiswal, and E. Speranza, Relativistic fluid dynamics with spin, Phys. Rev. C 97, 041901 (2018).

[17] W. Florkowski, B. Friman, A. Jaiswal, R. Ryblewski, and E. Speranza, Spin-dependent distribution functions for relativistic hydrodynamics of spin-1/2 particles, Phys. Rev. D 97, 116017 (2018).

[18] W. Florkowski, E. Speranza, and F. Becattini, Perfect-fluid hydrodynamics with constant acceleration along the stream lines and spin polarization, Acta Phys. Pol. B 49, 1409 (2018).

[19] F. Becattini, W. Florkowski, and E. Speranza, Spin tensor and its role in non-equilibrium thermodynamics, Phys. Lett. B 789, 419 (2019).

[20] W. Florkowski, A. Kumar, and R. Ryblewski, Thermodynamic versus kinetic approach to polarization-vorticity coupling, Phys. Rev. C 98, 044906 (2018).

[21] W. Florkowski, A. Kumar, R. Ryblewski, and A. Mazeliauskas, Longitudinal spin polarization in a thermal model, Phys. Rev. C 100, 054907 (2019).

[22] W. Florkowski, A. Kumar, R. Ryblewski, and R. Singh, Spin polarization evolution in a boost invariant hydrodynamical background, Phys. Rev. C 99, 044910 (2019).

[23] S. Bhadury, W. Florkowski, A. Jaiswal, A. Kumar, and R. Ryblewski, Relativistic dissipative spin dynamics in the relaxation time approximation, Phys. Lett. B 814, 136096 (2021).

[24] S. Bhadury, W. Florkowski, A. Jaiswal, A. Kumar, and R. Ryblewski, Dissipative spin dynamics in relativistic matter, Phys. Rev. D 103, 014030 (2021).

[25] W. Florkowski, R. Ryblewski, and A. Kumar, Relativistic hydrodynamics for spin-polarized fluids, Prog. Part. Nucl. Phys. 108, 103709 (2019).

[26] L. Tinti and W. Florkowski, Particle polarization, spin tensor and the Wigner distribution in relativistic systems, arXiv:2007.04029.

[27] F. Becattini, V. Chandra, L. Del Zanna, and E. Grossi, Relativistic distribution function for particles with spin at local thermodynamical equilibrium, Ann. Phys. (Amsterdam) 338, 32 (2013).

[28] F. Becattini, L. Csernai, and D. J. Wang, $\Lambda$ polarization in peripheral heavy ion collisions, Phys. Rev. C 88, 034905 (2013); , Erratum, Phys. Rev. C 93, 069901 (2016).

[29] F. Becattini, I. Karpenko, M. Lisa, I. Upsal, and S. Voloshin, Global hyperon polarization at local thermodynamic equilibrium with vorticity, magnetic field and feeddown, Phys. Rev. C 95, 054902 (2017).

[30] I. Karpenko and F. Becattini, Study of $\Lambda$ polarization in relativistic nuclear collisions at $\sqrt{s_{\mathrm{NN}}}=7.7200 \mathrm{GeV}$, Eur. Phys. J. C 77, 213 (2017).

[31] Y. Xie, D. Wang, and L. P. Csernai, Global $\Lambda$ polarization in high energy collisions, Phys. Rev. C 95, 031901 (2017).

[32] Y. Sun and C. M. Ko, $\Lambda$ hyperon polarization in relativistic heavy ion collisions from a chiral kinetic approach, Phys. Rev. C 96, 024906 (2017).

[33] H. Li, L.-G. Pang, Q. Wang, and X.-L. Xia, Global $\Lambda$ polarization in heavy-ion collisions from a transport model, Phys. Rev. C 96, 054908 (2017).

[34] F. Becattini and I. Karpenko, Collective Longitudinal Polarization in Relativistic Heavy-Ion Collisions at Very High Energy, Phys. Rev. Lett. 120, 012302 (2018).

[35] D.-X. Wei, W.-T. Deng, and X.-G. Huang, Thermal vorticity and spin polarization in heavy-ion collisions, Phys. Rev. C 99, 014905 (2019).

[36] X.-L. Xia, H. Li, Z.-B. Tang, and Q. Wang, Probing vorticity structure in heavy-ion collisions by local $\Lambda$ polarization, Phys. Rev. C 98, 024905 (2018). 
[37] Y. Sun and C. M. Ko, Azimuthal angle dependence of the longitudinal spin polarization in relativistic heavy ion collisions, Phys. Rev. C 99, 011903 (2019).

[38] Y. B. Ivanov, V. Toneev, and A. Soldatov, Vorticity and particle polarization in relativistic heavy-ion collisions, Phys. At. Nucl. 83, 179 (2020).

[39] F. Becattini, G. Cao, and E. Speranza, Polarization transfer in hyperon decays and its effect in relativistic nuclear collisions, Eur. Phys. J. C 79, 741 (2019).

[40] J.-j. Zhang, R.-h. Fang, Q. Wang, and X.-N. Wang, A microscopic description for polarization in particle scatterings, Phys. Rev. C 100, 064904 (2019).

[41] J. I. Kapusta, E. Rrapaj, and S. Rudaz, Hyperon polarization in relativistic heavy ion collisions and axial U(1) symmetry breaking at high temperature, Phys. Rev. C 101, 031901 (2020).

[42] Z. Wang, X. Guo, and P. Zhuang, Local equilibrium spin distribution from detailed balance, arXiv:2009.10930.

[43] K. Fukushima and S. Pu, Spin Hydrodynamics and symmetric energy-momentum tensors-A current induced by the spin vorticity, arXiv:2010.01608.

[44] B. Fu, K. Xu, X.-G. Huang, and H. Song, A hydrodynamic study of hyperon spin polarization in relativistic heavy ion collisions, Phys. Rev. C 103, 024903 (2021).

[45] J.-H. Gao, Z.-T. Liang, Q. Wang, and X.-N. Wang, Global polarization effect and spin-orbit coupling in strong interaction, arXiv:2009.04803.

[46] J.-H. Gao, Z.-T. Liang, and Q. Wang, Quantum kinetic theory for spin-1/2 fermions in Wigner function formalism, Int. J. Mod. Phys. A 36, 2130001 (2021).

[47] X.-G. Huang, J. Liao, Q. Wang, and X.-L. Xia, Vorticity and spin polarization in heavy ion collisions: Transport models, arXiv:2010.08937.

[48] S. A. Voloshin, Polarized secondary particles in unpolarized high energy hadron-hadron collisions?, arXiv:nucl-th/ 0410089.

[49] B. Betz, M. Gyulassy, and G. Torrieri, Polarization probes of vorticity in heavy ion collisions, Phys. Rev. C 76, 044901 (2007).

[50] A. Avkhadiev and A. V. Sadofyev, Chiral vortical effect for bosons, Phys. Rev. D 96, 045015 (2017).

[51] M. Baznat, K. Gudima, A. Sorin, and O. Teryaev, Hyperon polarization in heavy-ion collisions and holographic gravitational anomaly, Phys. Rev. C 97, 041902 (2018).

[52] D. Montenegro, L. Tinti, and G. Torrieri, The ideal relativistic fluid limit for a medium with polarization, Phys. Rev. D 96, 056012 (2017).

[53] D. Montenegro and G. Torrieri, Causality and dissipation in relativistic polarizable fluids, Phys. Rev. D 100, 056011 (2019).

[54] K. Fukushima, Extreme matter in electromagnetic fields and rotation, Prog. Part. Nucl. Phys. 107, 167 (2019).

[55] G. Y. Prokhorov, O. V. Teryaev, and V. I. Zakharov, Effects of rotation and acceleration in the axial current: Density operator vs Wigner function, J. High Energy Phys. 02 (2019) 146.

[56] B. Mcinnes, Holography of low-centrality heavy ion collisions, Int. J. Mod. Phys. A 34, 1950174 (2019).

[57] X.-G. Huang and A. V. Sadofyev, Chiral vortical effect for an arbitrary spin, J. High Energy Phys. 03 (2019) 084.
[58] D.-L. Yang, Side-jump induced spin-orbit interaction of chiral fluids from kinetic theory, Phys. Rev. D 98, 076019 (2018).

[59] X.-L. Xia, H. Li, X.-G. Huang, and H. Z. Huang, Feeddown effect on $\Lambda$ spin polarization, Phys. Rev. C 100, 014913 (2019).

[60] S. Li and H.-U. Yee, Quantum kinetic theory of spin polarization of massive quarks in perturbative QCD: Leading log, Phys. Rev. D 100, 056022 (2019).

[61] J. I. Kapusta, E. Rrapaj, and S. Rudaz, Relaxation Time for Strange Quark Spin in Rotating Quark-Gluon Plasma, Phys. Rev. C 101, 024907 (2020).

[62] S. Y. Liu, Y. Sun, and C. M. Ko, Spin Polarizations in a Covariant Angular-Momentum-Conserved Chiral Transport Model, Phys. Rev. Lett. 125, 062301 (2020).

[63] Y. B. Ivanov, V. Toneev, and A. Soldatov, Estimates of hyperon polarization in heavy-ion collisions at collision energies $\sqrt{s_{N N}}=4-40 \mathrm{GeV}$, Phys. Rev. C 100, 014908 (2019).

[64] J. Zhao and F. Wang, Experimental searches for the chiral magnetic effect in heavy-ion collisions, Prog. Part. Nucl. Phys. 107, 200 (2019).

[65] V.E. Ambrus, Helical massive fermions under rotation, J. High Energy Phys. 08 (2020) 016.

[66] X.-L. Sheng, L. Oliva, and Q. Wang, What can we learn from the global spin alignment of $\phi$ mesons in heavy-ion collisions?, Phys. Rev. D 101, 096005 (2020).

[67] J.-H. Gao and Z.-T. Liang, Relativistic quantum kinetic theory for massive fermions and spin effects, Phys. Rev. D 100, 056021 (2019).

[68] K. Hattori, M. Hongo, X.-G. Huang, M. Matsuo, and H. Taya, Fate of spin polarization in a relativistic fluid: An entropy-current analysis, Phys. Lett. B 795, 100 (2019).

[69] G. Y. Prokhorov, O. V. Teryaev, and V. I. Zakharov, Unruh effect universality: Emergent conical geometry from density operator, J. High Energy Phys. 03 (2020) 137.

[70] G. Y. Prokhorov, O. V. Teryaev, and V. I. Zakharov, Thermodynamics of accelerated fermion gases and their instability at the Unruh temperature, Phys. Rev. D 100, 125009 (2019).

[71] A. Freese and I. C. Cloët, Gravitational form factors of light mesons, Phys. Rev. C 100, 015201 (2019).

[72] A. Huang, S. Shi, X. Zhu, L. He, J. Liao, and P. Zhuang, The quantum kinetic equation and dynamical mass generation in $2+1$ dimensions, arXiv:2007.02858 [Phys. Rev. D (to be published)].

[73] X. Guo, Massless limit of transport theory for massive fermions, Chin. Phys. C 44, 104106 (2020).

[74] G. Prokhorov, O. Teryaev, and V. Zakharov, CVE for photons: Black-hole vs. flat-space derivation, Phys. Rev. D 102, 121702 (2020).

[75] F. Becattini, M. Buzzegoli, and A. Palermo, Exact equilibrium distributions in statistical quantum field theory with rotation and acceleration: Scalar field, J. High Energy Phys. 02 (2021) 101.

[76] F. Becattini, M. Buzzegoli, A. Palermo, and G. Prokhorov, Polarization as a signature of local parity violation in hot QCD matter, arXiv:2009.13449. 
[77] Y. Ivanov and A. Soldatov, Correlation between global polarization, angular momentum, and flow in heavy-ion collisions, Phys. Rev. C 102, 024916 (2020).

[78] X.-G. Deng, X.-G. Huang, Y.-G. Ma, and S. Zhang, Vorticity in low-energy heavy-ion collisions, Phys. Rev. C 101, 064908 (2020).

[79] D. Hou and S. Lin, Polarization rotation of chiral fermions in vortical fluid, arXiv:2008.03862.

[80] D.-L. Yang, K. Hattori, and Y. Hidaka, Effective quantum kinetic theory for spin transport of fermions with collsional effects, J. High Energy Phys. 07 (2020) 070.

[81] A. D. Gallegos and U. Gürsoy, Holographic spin liquids and Lovelock Chern-Simons gravity, J. High Energy Phys. 11 (2020) 151.

[82] M. Kawaguchi, S. Matsuzaki, and X.-G. Huang, Dynamic scale anomalous transport in QCD with electromagnetic background, J. High Energy Phys. 10 (2020) 017.

[83] W. Li and G. Wang, Chiral magnetic effects in nuclear collisions, Annu. Rev. Nucl. Part. Sci. 70, 293 (2020).

[84] S. Li, M. A. Stephanov, and H.-U. Yee, Non-dissipative second-order transport, spin, and pseudo-gauge transformations in hydrodynamics, arXiv:2011.12318.

[85] K. Hattori, Y. Hidaka, N. Yamamoto, and D.-L. Yang, Wigner functions and quantum kinetic theory of polarized photons, J. High Energy Phys. 02 (2021) 001.

[86] S. Shi, C. Gale, and S. Jeon, Relativistic viscous spin hydrodynamics from chiral kinetic theory, arXiv:2008 .08618 .

[87] M. Garbiso and M. Kaminski, Hydrodynamics of simply spinning black holes \& hydrodynamics for spinning quantum fluids, J. High Energy Phys. 12 (2020) 112.

[88] X.-G. Huang, P. Mitkin, A. V. Sadofyev, and E. Speranza, Zilch vortical effect, berry phase, and kinetic theory, J. High Energy Phys. 10 (2020) 117.

[89] A. D. Gallegos, U. Gürsoy, and A. Yarom, Hydrodynamics of spin currents, arXiv:2101.04759.

[90] F. Becattini and F. Piccinini, The ideal relativistic spinning gas: Polarization and spectra, Ann. Phys. (Amsterdam) 323, 2452 (2008).

[91] F. Becattini and L. Tinti, The Ideal relativistic rotating gas as a perfect fluid with spin, Ann. Phys. (Amsterdam) 325, 1566 (2010).

[92] F. Becattini and E. Grossi, Quantum corrections to the stress-energy tensor in thermodynamic equilibrium with acceleration, Phys. Rev. D 92, 045037 (2015).

[93] S. S. Gubser, Symmetry constraints on generalizations of Bjorken flow, Phys. Rev. D 82, 085027 (2010).

[94] S. S. Gubser and A. Yarom, Conformal hydrodynamics in Minkowski and de Sitter spacetimes, Nucl. Phys. B846, 469 (2011).

[95] G. S. Denicol, U. W. Heinz, M. Martinez, J. Noronha, and M. Strickland, New Exact Solution of the Relativistic Boltzmann Equation and its Hydrodynamic Limit, Phys. Rev. Lett. 113, 202301 (2014).

[96] M. Nopoush, R. Ryblewski, and M. Strickland, Anisotropic hydrodynamics for conformal Gubser flow, Phys. Rev. D 91, 045007 (2015).

[97] M. Nopoush, M. Strickland, R. Ryblewski, D. Bazow, U. Heinz, and M. Martinez, Leading-order anisotropic hydrodynamics for central collisions, Phys. Rev. C 92, 044912 (2015).

[98] M. Martinez, M. McNelis, and U. Heinz, Anisotropic fluid dynamics for Gubser flow, Phys. Rev. C 95, 054907 (2017).

[99] C. Chattopadhyay, U. Heinz, S. Pal, and G. Vujanovic, Higher order and anisotropic hydrodynamics for Bjorken and Gubser flows, Phys. Rev. C 97, 064909 (2018).

[100] E. Calzetta and L. Cantarutti, Dissipative type theories for Bjorken and Gubser flows, Int. J. Mod. Phys. A 35, 2050074 (2020).

[101] A. Behtash, S. Kamata, M. Martinez, and H. Shi, Global flow structure and exact formal transseries of the Gubser flow in kinetic theory, J. High Energy Phys. 07 (2020) 226.

[102] A. Dash and V. Roy, Hydrodynamic attractors for Gubser flow, Phys. Lett. B 806, 135481 (2020).

[103] Z. F. Jiang, D. She, C. Yang, and D. Hou, Perturbation solutions of relativistic viscous hydrodynamics for longitudinally expanding fireballs, Chin. Phys. C 44, 084107 (2020).

[104] M. Shokri and F. Taghinavaz, Bjorken flow in the general frame and its attractor, Chin. Phys. C 44, 084107 (2020).

[105] S. De Groot, Relativistic Kinetic Theory. Principles and Applications (North-Holland, New York, 1980), https:// inspirehep.net/literature/162065.

[106] R. Baier, P. Romatschke, D. T. Son, A. O. Starinets, and M. A. Stephanov, Relativistic viscous hydrodynamics, conformal invariance, and holography, J. High Energy Phys. 04 (2008) 100.

[107] S. Bhattacharyya, S. Lahiri, R. Loganayagam, and S. Minwalla, Large rotating AdS black holes from fluid mechanics, J. High Energy Phys. 09 (2008) 054.

[108] R. Loganayagam, Entropy current in conformal hydrodynamics, J. High Energy Phys. 05 (2008) 087.

[109] S. Weinberg, The Quantum Theory of Fields. Vol. 1: Foundations (Cambridge University Press, Cambridge, England, 2005).

[110] E. Speranza and N. Weickgenannt, Spin tensor and pseudo-gauges: From nuclear collisions to gravitational physics, arXiv:2007.00138.

[111] N. Weickgenannt, E. Speranza, X.-1. Sheng, Q. Wang, and D. H. Rischke, Generating spin polarization from vorticity through nonlocal collisions, arXiv:2005.01506.

[112] H. Kastrup, On the advancements of conformal transformations and their associated symmetries in geometry and theoretical physics, Ann. Phys. (Amsterdam) 17, 631 (2008).

[113] L. Fabbri, Conformal gravity with Dirac matter, Ann. Fond. Louis de Broglie 38, 155 (2013), arXiv: 1101.2334.

[114] R. M. Wald, General Relativity (Chicago University Press, Chicago, IL, 1984), https://cds.cern.ch/record/106274.

[115] V. Faraoni, E. Gunzig, and P. Nardone, Conformal transformations in classical gravitational theories and in cosmology, Fundam. Cosm. Phys. 20, 121 (1999), arXiv: gr-qc/9811047. 
[116] J. Callan, G. Curtis, S. R. Coleman, and R. Jackiw, A new improved energy-momentum tensor, Ann. Phys. (N.Y.) 59, 42 (1970).

[117] J. Wess, Conformal invariance and the energy-momentum tensor, Springer Tracts Mod. Phys. 60, 1 (1971).

[118] P. Di Francesco, P. Mathieu, and D. Senechal, Conformal Field Theory, Graduate Texts in Contemporary Physics (Springer-Verlag, New York, 1997).

[119] M. Forger and H. Romer, Currents and the energy momentum tensor in classical field theory: A fresh look at an old problem, Ann. Phys. (Amsterdam) 309, 306 (2004).
[120] L. Du, U. Heinz, K. Rajagopal, and Y. Yin, Fluctuation dynamics near the QCD critical point, Phys. Rev. C 102, 054911 (2020).

[121] H. Marrochio, J. Noronha, G. S. Denicol, M. Luzum, S. Jeon, and C. Gale, Solutions of conformal Israel-Stewart relativistic viscous fluid dynamics, Phys. Rev. C 91, 014903 (2015).

[122] R. C. Fernow and A. Krisch, High-energy Physics with polarized proton beams, Annu. Rev. Nucl. Part. Sci. 31, 107 (1981).

[123] R. G. Milner, A short history of spin, Proc. Sci., PSTP2013 (2013) 003 [arXiv:1311.5016]. 